\title{
Scheduler Modeling Based on the Controller Synthesis Paradigm
}

\author{
Joseph Sifakis \\ VERIMAG \\ 2 Av. de Vignate \\ 38610 Gières, France \\ Joseph.Sifakis@imag.fr
}

\begin{abstract}
The controller synthesis paradigm provides a general framework for scheduling real-time applications. Schedulers can be considered as controllers of the applications; they restrict their behavior so that given scheduling requirements are met.

We study a modeling methodology based on the controller synthesis paradigm. The methodology allows to get a correctly scheduled system from timed models of its processes, in an incremental manner, by application of composability results which simplify schedulability analysis. It consists in restricting successively the system to be scheduled by application of constraints defined from scheduling requirements. The latter are a conjunction of schedulability requirements that express timing properties of the processes and policy requirements about resource management. The presented methodology allows a unified view of analytic approaches and model-based approaches to scheduling.
\end{abstract}

